\title{
Effects of protoporphyrins on production of nitric oxide and expression of vascular endothelial growth factor in vascular smooth muscle cells and macrophages ${ }^{\star}$
}

\author{
Alicja Józkowicz ${ }^{1,3}$ and Józef Dulak ${ }^{2}$ \\ ${ }^{1}$ Laboratory of Molecular Genetics and Genetic Engineering, ${ }^{2}$ Department of Cell Biochemistry, \\ Faculty of Biotechnology, Jagiellonian University, Kraków, Poland; ${ }^{3}$ Department of Vascular \\ Surgery, University of Vienna, Austria
}

Received: 02 January, 2003; accepted: 11 February, 2003

Key words: metalloporphyrins, heme oxygenase, vascular endothelial growth factor, nitric oxide, cell viability

\begin{abstract}
Heme oxygenase-1 (HO-1), an inducible enzyme degrading heme to biliverdin, iron and carbon monoxide, is involved in regulation of inflammation and angiogenesis. Tin protoporphyrin (SnPPIX) and zinc protoporphyrin (ZnPPIX) are commonly used as competitive inhibitors of HO-1. We aimed to compare the effects of SnPPIX and ZnPPIX on the production of vascular endothelial growth factor (VEGF), activity of inducible nitric oxide synthase (iNOS) and cell viability. All experiments were performed on rat vascular smooth muscle cells and murine RAW264.7 macrophages treated with 3-10 $\mu \mathrm{M}$ protoporphyrins. Some cells were additionally stimulated with IL-1 $\beta$ or with lipopolysaccharide. After a $24 \mathrm{~h}$ incubation period SnPPIX and ZnPPIX significantly reduced the generation of VEGF in vascular smooth muscle cells and RAW264.7, both in resting and stimulated cells. The inhibitory potentials of both protoporphyrins on VEGF synthesis were very similar. In contrast, analysis of iNOS activity revealed that results obtained with different HO-1 inhibitors are discrepant.
\end{abstract}

\footnotetext{
${ }_{\text {This work was partially supported by grant from the State Committee for Scientific Research (KBN, Po- }}$ land) No. 3 P04A 04922 and grant from the Jagiellonian University, No. N-25/CRBW-VII-22/2002 awarded to J.D.

${ }^{\square}$ Corresponding author: Alicja Józkowicz, Laboratory of Molecular Genetics and Genetic Engineering, Faculty of Biotechnology, Jagiellonian University, Gronostajowa 7, 30-387 Kraków, Poland; tel.: (48 12) 252 6411; fax: (48 12) 252 6902; e-mail: alicia@mol.uj.edu.pl

Abbreviations: HOs, heme oxygenases; IFN- $\gamma$, interferon $-\gamma$; IL- $1 \beta$, interleukin- $1 \beta$; iNOS, inducible nitric oxide synthase; LDH, lactate dehydrogenase; LPS, lipopolysaccharide; CoPPIX, cobalt protoporphyrin-IX; CuPPIX, copper protoporphyrin-IX; SnMPIX, tin mesoporphyrin-IX; SnPPIX, tin protoporphyrin-IX; VEGF, vascular endothelial growth factor; VSMC, vascular smooth muscle cells; ZnDPIX, zinc deuteroporphyrin-IX; ZnMPIX, zinc mesoporphyrin-IX; ZnPPIX, zinc protoporphyrin-IX.
} 
Generation of nitric oxide by iNOS was significantly increased by SnPPIX but strongly decreased by ZnPPIX. Similar differences were observed when cell viability was compared. SnPPIX improved the cell survival rate, whereas the same doses of ZnPPIX exerted some cytotoxic effects. In summary, SnPPIX and ZnPPIX can be used as HO-1 inhibitors in some experimental models. However, these compounds produce also HO-independent effects, which can make the interpretation of experiments very uncertain. Thus the involvement of the HO-1 pathway should be always confirmed by more specific methods.

Heme oxygenases (HOs) are involved in the degradation of heme, resulting in the generation of biliverdin, iron and carbon monoxide. Three distinct variants of HOs have been described so far. HO-2 and HO-3 are constitutively expressed, whereas HO-1 can be potently induced by heme, prostaglandin- $\mathrm{J}_{2}$, oxidized lipoproteins, inflammatory cytokines, hypoxia, nitric oxide or heavy metals (Snyder \& Baranano, 2001; Józkowicz et al., 2002).

The last decade has brought a dramatic upsurge in the interest in biologic effects of HO-1 activity. Numerous studies have demonstrated that products of this enzymatic reaction have important anti-oxidant, anti-inflammatory or cytoprotective functions, and can modulate inter- and intracellular signaling mechanisms (for a review see: Snyder \& Baranano, 2001). In consequence, HO-1 has been shown to have salutary effects in pathological states as diverse as atherosclerosis, hypertension, sepsis, and transplant rejection (for a review see: Morse \& Choi, 2002).

The mechanisms by which HO-1 confers its protective effects are as yet poorly understood, but this area of investigations is active and rapidly evolving. The simplest and most commonly used pharmacological tools employed by researchers are metalloporphyrins, competitive inhibitors of HO-1.

The potency of metalloporphyrins as blockers of HO-1 is affected by the metal cation associated with the porphyrin ring as well as by different ring substituents (Vreman et al., 1993). Inhibition of HO-1 enzymatic activity has been demonstrated for zinc protoporphyrin-IX (ZnPPIX), zinc deuteroporphyrin-IX (ZnDPIX), zinc mesoporphyrin (ZnMPIX), tin protoporphyrin-IX (SnPPIX), and tin mesoporphyrin-IX (SnMPIX). At a concentration of $10 \mu \mathrm{M}$, the weakest inhibitor was ZnDPIX (30\% inhibition), whereas ZnPPIX, ZnMPIX, SnPPIX and SnMPIX decreased HO-1 activity by $60 \%-85 \%$ (Anderson et al., 1984; Appleton et al., 1999; Beri \& Chandra, 1993; Dulak et al., 2002a; Lutton et al., 1997; Vreman et al., 1993; Vreman et al., 1996; Weiss et al., 1993; Steffensrud, 1998). All these compounds are being widely used in studies performed in vitro to clarify the molecular pathways involved in HO-1 signal transduction and in experiments in vivo designed to establish the role of HO-1 or to prevent and treat hyperbilirubinemia (Zakhary et al., 1996; Grunemar \& Ny, 1997; Steffensrud, 1998; Doi et al., 1999; Cavicchi et al., 2000; Tamion et al., 2001). Finally, ZnPPIX, SnPPIX and SnMPIX have been exploited therapeutically to reduce jaundice in the neonates (Quato \& Maines, 1985; Anderson et al., 1986; Drummond, 1987).

Little is known, however, about the specificity of these inhibitors and some data identified important differences between the biological and toxicological properties of particular porphyrins. It has been shown that ZnPPIX, but not SnPPIX or ZnDPIX, applied to pituitary cells led to an irreversible attenuation of $\mathrm{Ca}^{2+}$ current (Linden et al., 1993). Likewise, ZnPPIX, but not SnPPIX, significantly inhibited hematopoiesis in rabbit and human bone marrow (Lutton et al., 1997). Even more striking differences were reported in experiments performed on monocytes, where ZnMPIX almost fully inhibited IFN- $\gamma$-induced degradation of tryptophan, whereas SnPPIX enhanced the IFN effects (Weiss et al., 1993). This lack of specificity of the heme oxygenase 
inhibiting metalloporphyrins impedes the interpretation of the obtained results.

Numerous studies have demonstrated that metalloporphyrins can modulate the expression or activity of mediators involved in angiogenesis and inflammation. In particular, they influence the production of nitric oxide (NO) by inducible nitric oxide synthase (iNOS) (Dulak et al., 1999; Cavicchi et al., 2000) and affect the generation of vascular endothelial growth factor (VEGF), one of the most prominent pro-angiogenic proteins (Dulak et al., 2002a; Józkowicz et al., 2002). They can also modulate the viability of cells (Lutton et al., 1997). In most reports, however, only one pharmacological HO-1 inhibitor was employed, thus some unspecific activities of the metalloporphyrin used could not be excluded.

Therefore, in the present study we decided to compare the effects of several metalloporphyrins on generation of VEGF, activity of iNOS and cell viability. To avoid a false generalization of possible cell-specific effects, we performed experiments on two distinct cell types, namely on rat vascular smooth muscle cells and on murine RAW264.7 macrophages.

\section{MATERIALS AND METHODS}

Chemicals. Interleukin- $1 \beta$ (IL-1 $\beta$ ) and lipopolysaccharide (LPS) were purchased from Sigma, culture media (DMEM F-12, RPMI-1641) and fetal calf serum (FCS) were from Gibco. CytoTox-96 assay and Griess Reagent System were bought from Promega. ELISA kit for mouse/rat VEGF was obtained from R\&D Systems. Tin protoporphyrin-IX (SnPPIX), copper protoporphyrin-IX (CuPPIX), zinc protoporphyrin-IX (ZnPPIX), zinc deuteroporphyrin-IX (ZnDPIX), and cobalt protoporphyrin-IX (CoPPIX) were purchased from PorphyrinProducts.

Cell culture. Murine macrophage-like cells RAW264.7 were cultured in RPMI-1641 medium supplemented with $10 \%$ FCS. Vascular smooth muscle cells (VSMC) were isolated by collagenase digestion of rat thoracic aorta and cultured in DMEM F-12 medium supplemented with $5 \%$ FCS. VSMC of 5 th to 10 th passages were used for the experiments. VSMC or RAW264.7 were seeded in a 24-well plate and grown to confluence. Before experiments, the cells were starved over night in medium containing $0.5 \%$ FCS. Then the medium was replaced with fresh one and cells were supplemented with protoporphyrins (1-10 $\mu \mathrm{M})$. Half an hour later, some VSMC and RAW264.7 were additionally stimulated with IL-1 $\beta$ ( $5 \mathrm{ng} / \mathrm{ml})$ or LPS $(100 \mathrm{ng} / \mathrm{ml})$, respectively. As we have demonstrated in earlier studies, these doses of IL- $1 \beta$ and LPS did not exert any cytotoxic effects on VSMC and RAW264.7 (Józkowicz et al., 2000). After a $24 \mathrm{~h}$ incubation period the culture media were harvested and frozen.

Measurement of VEGF protein concentrations. Concentrations of VEGF protein in the culture media were quantified using the sandwich ELISA, following the manufacturer's instructions.

Cell viability assay. Cell viability was assessed by colorimetric measurement of LDH release according to vendor's protocol.

Statistical analysis. All experiments were performed in duplicates or triplicates and were repeated at least 3 times. Data are presented as mean \pm E.M. Statistical evaluation was done with ANOVA followed by Tukey test. Differences were accepted as statistically significant at $P<0.05$.

\section{RESULTS}

\section{Effect of protoporphyrins on synthesis of VEGF}

The protoporphyrins SnPPIX and ZnPPIX are commonly used as competitive inhibitors of heme oxygenase-1 (HO-1) (Zakhary et al., 1996; Grunemar \& Ny, 1997; Doi et al., 1999; Cavicchi et al., 2000). To determine their ef- 

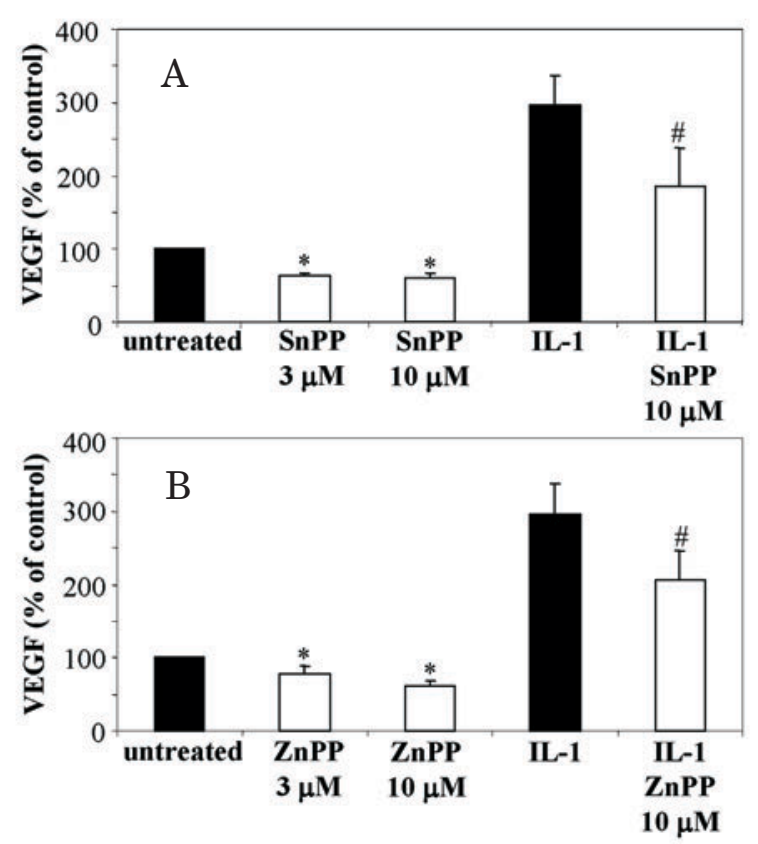

Figure 1. Effect of SnPPIX (A) and ZnPPIX (B) on release of VEGF from rat vascular smooth muscle cells.

Concentration of VEGF in culture medium was measured by ELISA. Each bar represents the mean \pm S.E.M. of 3-5 experiments, expressed as \% of control value (control, untreated cells). ${ }^{*} P<0.05$ in comparison to untreated cells, $\# P<0.05$ in comparison to IL- $1 \beta$-treated cells.

fects on the production of VEGF by rat VSMC and murine RAW264.7 we measured by ELISA the concentrations of VEGF protein in the culture media. After a $24 \mathrm{~h}$ incubation period, both types of cells released comparable amounts of VEGF, approx. 100-200 pg/ml. IL-1 $\beta$ significantly increased the generation of VEGF in VSMC (Fig. 1), while stimulation of RAW264.7 macrophages with LPS slightly inhibited the VEGF synthesis (Fig. 2).

In VSMC, SnPPIX (Fig. 1A) and ZnPPIX (Fig. 1B) significantly reduced the generation of VEGF both in resting and in IL- $1 \beta$-stimulated cells. The inhibitory potentials of both protoporphyrins were very similar. SnPPIX and ZnPPIX at doses of $10 \mu \mathrm{M}$ inhibited basal VEGF synthesis by $39.7 \%$ and $38.5 \%$, respectively. Similarly, the IL- $1 \beta$-stimulated VEGF was reduced by $37.6 \%$ and $30.1 \%$. Furthermore, SnPPIX and ZnPPIX strongly and dose-dependently attenuated the synthesis of
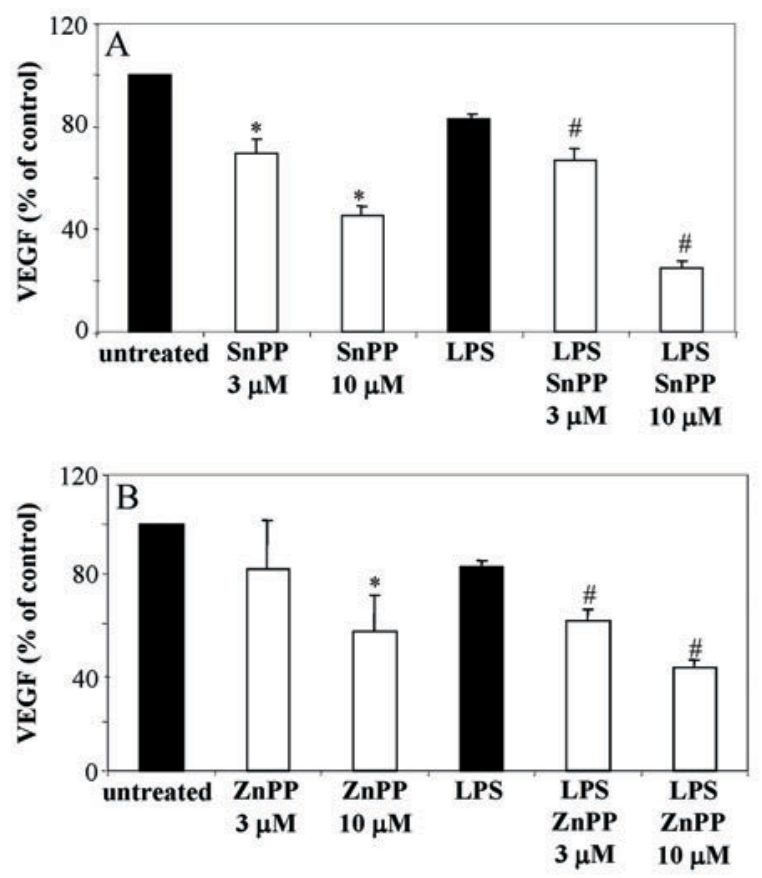

Figure 2. Effect of SnPPIX (A) and ZnPPIX (B) on release of VEGF from RAW264.7 murine macrophage-like cells.

Concentration of VEGF in culture medium was measured by ELISA. Each bar represents the mean \pm S.E.M. of 3-5 experiments, expressed as \% of control value (control, untreated cells). ${ }^{*} P<0.05$ in comparison to untreated cells, $\# P<0.05$ in comparison to LPS-treated cells.

VEGF in resting and LPS-stimulated RAW264.7 macrophages (Fig. 2A, B). Also in these cells the potencies of both HO-1 inhibitors were comparable. Noticeably, the same doses ( 3 and $10 \mu \mathrm{M}$ ) of CuPPIX, a protoporphyrin which does not influence the HO-1 activity (Chernick et al., 1989), did not affect the VEGF synthesis, while ZnDPIX, another potent inhibitor of HO-1, also attenuated VEGF synthesis (see: Dulak et al., 2002b; Józkowicz et al., 2002). This suggests that the observed decrease in the generation of VEGF in cells treated with SnPPIX or ZnPPIX may result from the inhibition of HO-1.

\section{Effect of protoporphyrins on iNOS activity}

Treatment of rat VSMC with $10 \mu \mathrm{M}$ SnPPIX increased strongly (by $54.6 \%$ ) the IL- $1 \beta$-stimulated activity of iNOS. Furthermore, it in- 

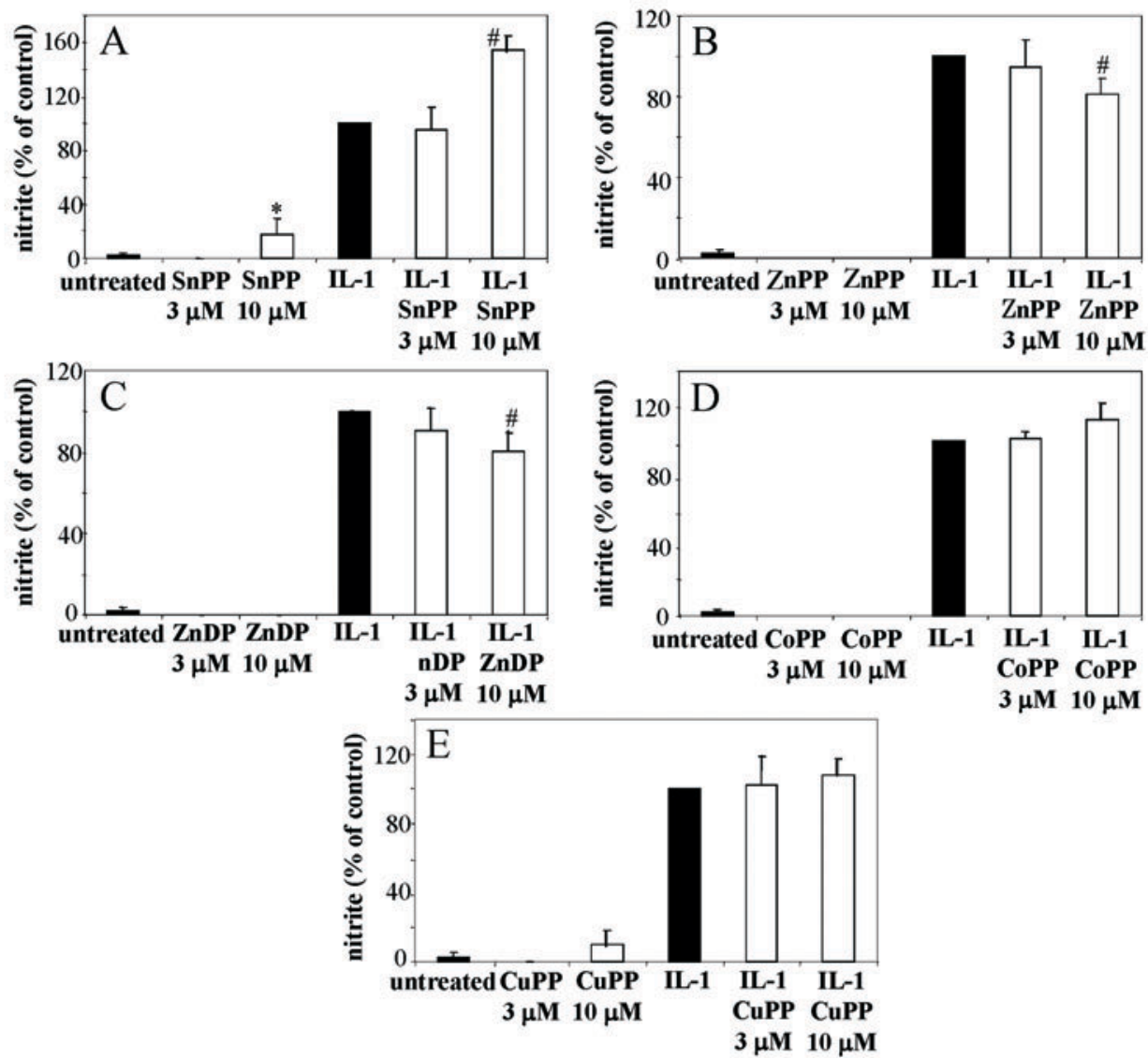

Figure 3. Effect of SnPPIX (A), ZnPPIX (B), ZnDPIX (C), CoPPIX (D), and CuPPIX (E) on production of NO by iNOS in rat vascular smooth muscle cells.

Generation of NO was assessed by measurement of nitrite concentration in culture media using Griess method. Each bar represents the mean \pm S.E.M. of 3-14 experiments, expressed as $\%$ of control value (control, IL-1 $\beta$-stimulated cells). ${ }^{*} P<0.05$ in comparison to untreated cells, $\# P<0.05$ in comparison to IL- $1 \beta$ treated cells. duced some nitrite accumulation in resting cells, not stimulated with IL- $1 \beta$ (Fig. 3A). The same effects, and even more pronounced, were observed in RAW264.7, where the treatment with $10 \mu \mathrm{M}$ SnPPIX resulted in three-fold enhancement of LPS-induced NO synthesis (Fig. 4). Surprisingly, the effects of ZnPPIX under the same experimental conditions were completely different. Incubation with $10 \mu \mathrm{M}$ ZnPPIX significantly decreased the synthesis of NO, reducing it by $20 \%$ and $45 \%$ in rat VSMC and RAW264.7, respectively (Figs. 3B and 4B). A similar decrease was observed in VSMC incubated with another zinc-containing protoporphyrin, ZnDPIX (Fig. 3C). CuPPIX did not influence significantly the generation of nitric oxide (Figs. 3E and 4D), whereas CoPPIX (protoporphyrin which induces HO-1 activity) did not change nitrite accumulation in VSMC, but strongly inhibited it in RAW264.7 macrophages (Figs. $3 \mathrm{D}$ and $4 \mathrm{C}$ ). This demonstrates that SnPPIX and $\mathrm{ZnPPIX}$ can exert distinct, non-specific ef- fects on iNOS, which are not associated with inhibition of HO-1.

\section{Effects of protoporphyrins on cell viability}

Analogous differences in the activities of SnPPIX and ZnPPIX were observed when cell viabilities were compared. SnPPIX improved significantly the cell survival rates both in rat VSMC and RAW264.7 (Figs. 5A and 6A), as assessed by colorimetric measurement of LDH activity in culture media. Treatment with SnPPIX reduced the release of LDH by $31.3 \%, 19.1 \%$ and $21.7 \%$ in non-stimulated VSMC, non-stimulated RAW264.7 and in LPS-activated RAW264.7, respectively. Only in VSMC stimulated with IL- $1 \beta$ such a protective effect of SnPPIX was not observed. In contrast, incubation of cells with ZnPPIX significantly decreased the cell viability. This cytotoxic effect was particularly pronounced in rat VSMC stimulated with cytokine (Fig. 5B) and in RAW264.7 activated with 

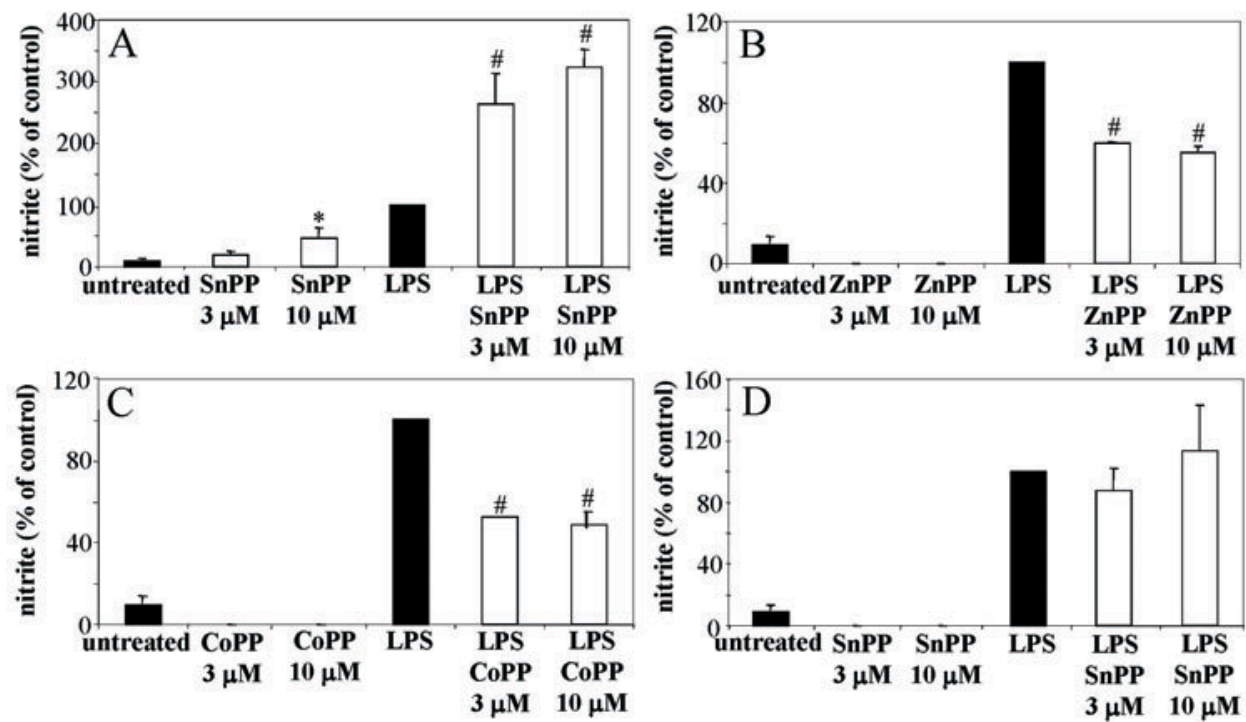

Figure 4. Effect of SnPPIX (A), ZnPPIX (B), CoPPIX (C), and CuPPIX (D) on production of NO by iNOS in RAW264.7 murine macrophage-like cells.

Generation of NO was assessed by measurement of nitrite concentration in culture media using Griess method. Each bar represents the mean \pm S.E.M. of 3-6 experiments, expressed as \% of control value (control, LPS-stimulated cells). ${ }^{*} P<0.05$ in comparison to untreated cells, $\# P<0.05$ in comparison to LPS-treated cells.
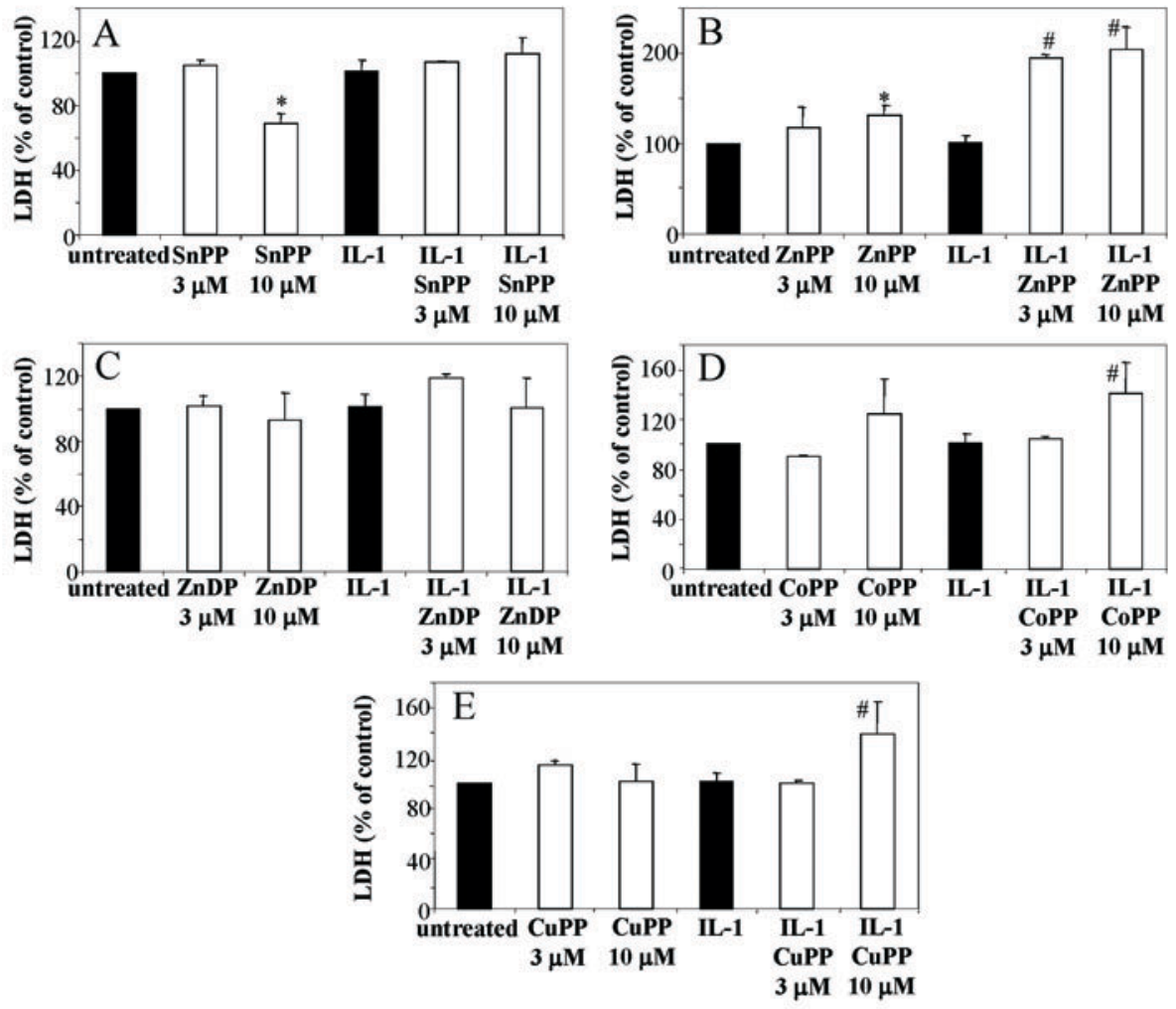

Figure 5. Effect of SnPPIX (A), ZnPPIX (B), ZnDPIX (C), CoPPIX (D), and CuPPIX (E) on viability of rat vascular smooth muscle cells.

The amount of dead cells was assessed by colorimetric measurement of LDH activity in culture medium. Each bar represents the mean \pm S.E.M. of 3-7 experiments, expressed as \% of control value (control, untreated cells). ${ }^{*} P<0.05$ in comparison to untreated cells, $\# P<0.05$ in comparison to IL- $1 \beta$-treated cells. 
endotoxin (Fig. 6B). Some cytotoxicity was also induced by CoPPIX and CuPPIX, whereas ZnDPIX did not affect the viability of the cells (Fig. 5C, D, E and Fig. 6C, D). Thus, like in the case of nitric oxide production, also
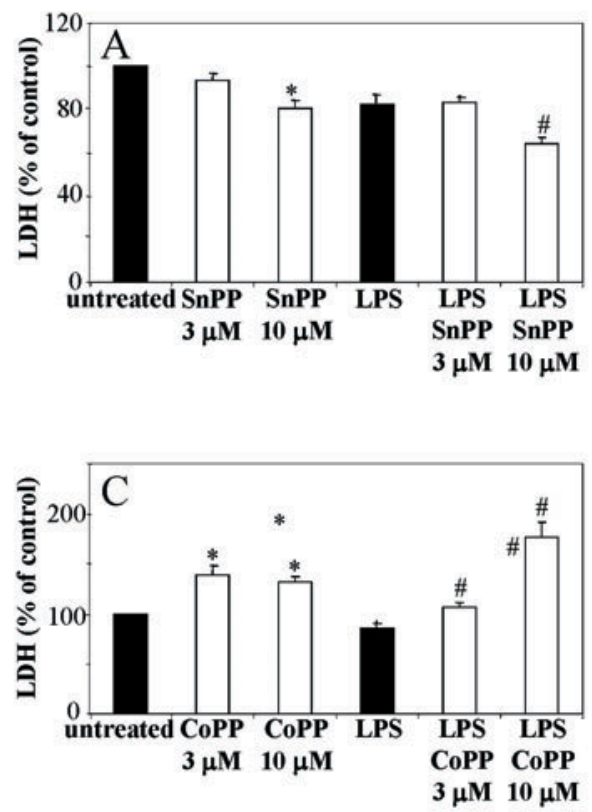

does not affect HO-1 (Chernick et al., 1989), does not change the VEGF expression (Dulak et al., 2002b; Józkowicz et al., 2002), our experiments strongly suggest that the effects of SnPPIX and ZnPPIX on VEGF production re-
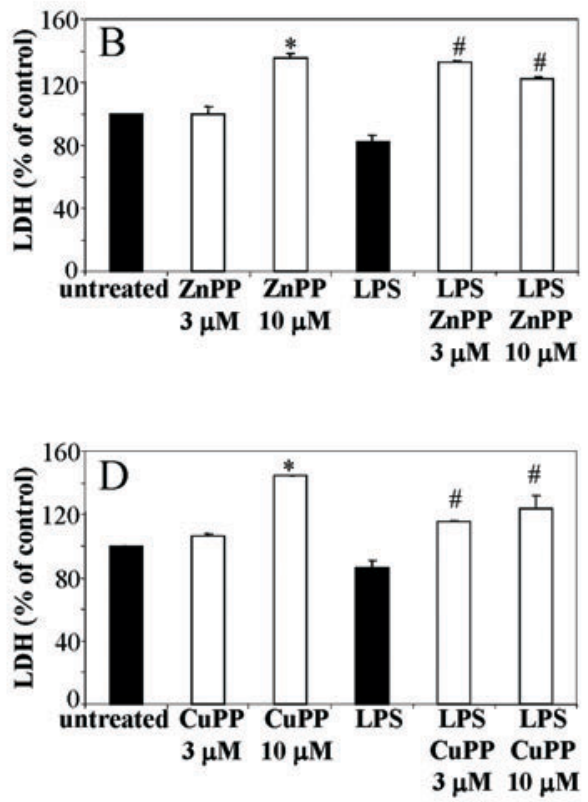

Figure 6. Effect of SnPPIX (A), ZnPPIX (B), ZnDPIX (C), and CoPPIX (D) on viability of RAW264.7 murine macrophage-like cells.

The amount of dead cells was assessed by colorimetric measurement of LDH activity in culture media. Each bar represents the mean \pm S.E.M. of 3 experiments, expressed as $\%$ of control value (control, untreated cells). ${ }^{*} P<0.05$ in comparison to untreated cells, $\# P<0.05$ in comparison to LPS-treated cells.

the effects of protoporphyrins on cell viability cannot be attributed solely to the modulation of HO-1 activity.

\section{DISCUSSION}

In previous papers we demonstrated that SnPPIX and ZnDPIX reduce the generation of VEGF in vascular smooth muscle cells (Dulak et al., 2002a; Dulak et al., 2002b) and in microvascular endothelial cells (Józkowicz et $a l ., 2002)$. Here we confirmed and extended these findings, demonstrating that two HO-1 blockers, SnPPIX and ZnPPIX, exert the same inhibitory influence on VEGF synthesis and that similar effects are displayed in VSMC and in macrophages. Together with the observation that $\mathrm{CuPPIX}$, a protoporphyrin which sult from the inhibition of heme oxygenase activity. This supposition is in concert with reports showing that pharmacological activation or genetical overexpression of HO-1 augment the synthesis of VEGF mRNA and protein in VSMC (Dulak et al., 2002a; Dulak et al., 2002b), microvascular endothelial cells (Józkowicz et al., 2002) and in placenta (Kreiser et al., 2002). This augmentation, caused by an increased transcription rate from the VEGF promoter, is possibly mediated by HO-generated carbon monoxide (Dulak et al., 2002a; Dulak et al., 2002b).

In contrast to the impact on the synthesis of VEGF, the influence of SnPPIX and ZnPPIX on nitric oxide production is strongly disturbed by their HO-independent actions. In fact, the effects of these HO-1 inhibitors on iNOS activity are opposite. SnPPIX signifi- 
cantly augments generation of NO both in IL- $1 \beta$-stimulated VSMC and in LPS-stimulated macrophages. In contrast, ZnPPIX under the same experimental conditions down-regulates the activity of iNOS. Interestingly, a similar level of inhibition is observed when VSMC are treated with another zinccontaining HO-1 inhibitor, ZnDPIX. Finally, the effects of CoPPIX, a HO-1 activator, are cell type-dependent: CoPPIX does not influence nitrite generation in VSMC, but inhibits it in RAW264.7.

It is well-known that both endogenous and exogenous NO induce the expression of HO-1 (Foresti et al. 1997; Doi et al., 1999; Polte et al., 2000; Kampfer et al., 2001). The reverse relation, that is the effect of HO-1 activation on nitric oxide production is less clear, although some findings suggest the mutual interplay between the iNOS and HO-1 systems (Dulak et al., 1999; Cavicchi et al., 2000). Thus it was supposed that HO-1-generated carbon monoxide can bind the heme prosthetic group, thereby inhibiting nitric oxide synthase function. Additionally, high activity of HO-1 could lead to shortage of heme, the cofactor necessary for NO synthases (White \& Marletta, 1992). In fact, it was shown that the heme-mediated induction of HO-1 in human intestinal epithelial cells or in murine RAW264.7 macrophages inhibits cytokine-induced iNOS activity (Wolff et al., 1996; Cavicchi et al., 2000). This inhibitory effect was abolished by SnPPIX (Cavicchi et al., 2000). However, when zinc porphyrin was used as an inhibitor of HO-1, the effect of HO-1 induction could not be reversed, but instead, ZnPPIX further inhibited iNOS (Wolff et al., 1996). These apparently contradictory results are in full agreement with our findings. On the basis of a direct comparison of the effects of SnPPIX and ZnPPIX we postulate that the influence of the inhibition of the HO-1 enzymatic activity are masked by HO-1 independent effects of metalloporphyrins on nitric oxide synthase. Taken together, our results reveal that protoporphyrins which mod- ulate HO-1 activity are not a suitable tool to investigate the role of the HO-1 pathway in nitric oxide production.

Some of those HO-independent actions could be associated with cytotoxicity of metalloporphyrins. For example, CoPPIX does not exert any cytotoxic effect and does not influence nitrite generation in rat VSMC, whereas it reduces cell viability and decreases $\mathrm{NO}$ production in murine RAW264.7 cultures. However, ZnDPIX, which strongly inhibits iNOS activity in VSMC, is not cytotoxic. Correspondingly, CuPPIX, which demonstrates some toxicity to RAW264.7, does not reduce NO generation in these cells, showing that not all inhibitory effects can be explained simply by the influence of protoporphyrins on cell survival.

Our experiments demonstrate also that, in contrast to other protoporphyrins, SnPPIX enhances the survival rate of VSMC and RAW264.7. A similar improvement of cell viability was found in human umbilical vein endothelial cells, human microvascular endothelial cells or murine fibroblasts, indicating that the protective effect of SnPPIX is cell-type independent (not shown). It cannot be also interpreted as a result of HO-1 inhibition, as other HO-1 blockers exert dissimilar effects: ZnDPIX does not influence the cell survival rate, whereas $\mathrm{ZnPPIX}$ at the same doses is cytotoxic for VSMC and RAW264.7, especially when cells are additionally stimulated with IL- $1 \beta$ or LPS.

A similar difference in the cytotoxicity of SnPPIX and ZnPPIX was reported by Lutton et al. (1997), who found that ZnPPIX was toxic for bone marrow cells, whereas SnPPIX did not show any significant influence on hematopoietic cell viability. Their and our data strongly confirm that the nature of the central metal atom in heme analogues profoundly affects the biological properties of metalloporphyrins. Tin incorporated in the porphyrin ring structure of SnPPIX is extremely stable and there is no known physiological mechanism by which the element can be removed from the porphyrin complex. In 
contrast, zinc porphyrins are highly labile in this regard (Lutton et al., 1997). Thus it was postulated that their cytotoxicity may be in part due to the release of zinc atom from the porphyrin ring, thus permitting the porphyrin macrocycle to exert a direct toxic effect on the cells (Greenbaum \& Kappas, 1991; Lutton et al., 1997). Also, zinc itself can substantially alter normal cell functions, leading to inhibition of iNOS activity (Sakaguchi et al., 2002) and increasing cell death rate (Koh et al., 1996; Lutton et al., 1997).

On the other hand, inhibition of HO-1 might additionally contribute to the ZnPPIX toxicity. It has been shown that blocking HO-1 by protoporphyrins increases sensitivity of rat artery smooth muscle cells to the injury evoked by hemin (Marton et al., 2000), augments cytotoxic effects of TNF- $\alpha$ in endothelial cells (Polte et al., 1997) and enhances mortality of RAW264.7 macrophages induced by UV-irradiation (Chen et al., 2002). Inhibition of HO-1 reduces also the viability of murine glial cells exposed to hemoglobin (Regan et al., 2000) and increases sensitivity of murine motor neurons to high doses of nitric oxide (Bishop et al., 1999). Regarding those reports, the protective effect of SnPPIX in our experiments is surprising and the mechanisms by which SnPPIX improves cell viability are not established. Because the increased survival rate was observed primarily in resting cells, which display a low activity of heme oxygenase, one can suppose that the protective effect of SnPPIX is independent of the HO-1 pathway.

The mechanisms responsible for the HO-independent activities of metalloporphyrins are not well recognized. It was demonstrated, for instance, that some effects are mediated by oxidative stress and can be reversed by co-administration of antioxidants (Linden et al., 1993; Chandra et al., 2000). Finally, ZnPPIX, ZnDPIX and SnPPIX may directly inhibit soluble guanylyl cyclase activity and down-regulate cGMP production (Luo \& Vincent, 1994; Serfass \& Burstyn, 1998).
In summary, a direct comparison of the effects of SnPPIX and ZnPPIX indicates that these metalloporphyrins can be used as HO-1 inhibitors in some experimental models, e.g. in studies on regulation of VEGF synthesis, as all compounds studied showed consistent results. However, the observed effects are also associated with a range of HO-independent actions, which can make the interpretation of experiments very uncertain. Thus the involvement of the HO-1 pathway should be always confirmed by more specific methods.

\section{R E F E R E N C E S}

Anderson KE, Simionatto CS, Drummond GS, Kappas A. (1984) Tissue distribution of tin protoporphyrin, a potent competitive inhibitor of heme oxygenase. $J$ Pharmacol Exp Ther:; 228: 327-33.

Anderson KE, Simionatto CS, Drummond GS, Kappas A. (1986) Disposition of tin-protoporphyrin and suppression of hyperbilirubinemia in humans. Clin Pharmacol Ther.; 39: 510-20.

Appleton SD, Chretien ML, McLaughlin BE, Vreman HJ, Stevenson DK, Brien JF, Nakaatsu K, Maurice DH, Marks GS. (1999) Selective inhibition of heme oxygenase, without inhibition of nitric oxide synthase or soluble guanylyl cyclase by metalloporphyrins at low concentrations. Drug Metab Dispos.; 27: 1214-9.

Beri R, Chandra R. (1993) Chemistry and biology of heme. Effect of metal salts, organometals, and metalloporphyrins on heme synthesis and catabolism, with special reference to clinical implications and interactions with cytochrome P-450. Drug Metab Rev.; 25: 149-52.

Bishop A, Marquis JC, Cashman NR, Demple B. (1999) Adaptive resistance to nitric oxide in motor neurons. Free Radical Biol Med.; 26: 978-86.

Cavicchi M, Gibbs L, Whittle BJ. (2000) Inhibition of inducible nitric oxide synthase in the human intestinal epithelial cell line, DLD-1, 
by the inducers of heme oxygenase 1 , bismuth salts, heme and nitric oxide donors. Gut.; 47: 771-8.

Chandra R, Upadhyaya G, Dass SK, Jain R. (2000) Co-administration of melatonin reverses the tin-protoporphyrin ( $\mathrm{SnPP}$ ) induced decline of cytochrome $\mathrm{P} 450$ content in vivo in rats. Eur J Drug Metab Pharmacokinet.; 25: $213-8$.

Chen YC, Shen SC, Lee WR, Lin HY, Ko CK, Lee TJ. (2002) Nitric oxide and prostaglandin E2 participate in lipopolysaccharide/interferon-gamma-induced heme oxygenase- 1 and prevent RAW264.7 macrophages from UV-irradiation-induced cell death. $J$ Cell Biochem.; 86: 331-9.

Chernick RJ, Martasek P, Levere RD, Margreiter R, Abraham NG. (1989) Sensitivity of human tissue heme oxygenase to a new synthetic metalloporphyrin. Hepatology.; 10: 365-9.

Doi K, Akaike T, Fujii S, Tanaka S, Ikebe N, Beppu T, Shibahara S, Ogawa M, Maeda H. (1999) Induction of heme oxygenase-1, nitric oxide and ischemia in experimental solid tumors and implication for tumor growth. $\mathrm{Br} J$ Cancer.; 80: 1945-54.

Drummond GS. (1987) Control of heme oxygenase metabolism by synthetic metalloporphyrins. Ann NY Acad Sci.; 514: 87-95.

Dulak J, Józkowicz A, Foresti R, Green C, Motterlini R. (1999) Regulation of nitric oxide synthesis by heme oxygenase- 1 in rat vascular smooth muscle cells. In Nitric oxide biology, part VII. Moncada S, Gustafsson L, Wiklund P, Higgs EA, eds, p 135. Portland Press, New York.

Dulak J, Józkowicz A, Foresti R, Kasza A, Frick M, Huk I, Green CJ, Pachinger O, Weidinger F, Motterlini R. (2002a) Heme oxygenase activity modulates vascular endothelial growth factor synthesis in vascular smooth muscle cells. Antioxidant Redox Signal.; 4: 229-40.

Dulak J, Motterlini R, Huk I, Pachinger O, Weidinger F, Jozkowicz A. (2002b) Carbon monoxide and iron, by-products of heme oxygenase, modulate vascular endothelial growth factor synthesis in vascular smooth muscle cells. In: Physiology and pathology of heme oxygenase. Abraham N, Alam J, Nath K, eds, pp 97-107. Kluwer Academic/Plenum Publishers, New York.

Foresti R, Clark JE, Green CJ, Motterlini RJ. (1997) Thiol compounds interact with nitric oxide in regulating heme oxygenase- 1 induction in endothelial cells. Involvement of superoxide and peroxynitrite anions. $J$ Biol Chem.; 272: 18411-7.

Greenbaum NL, Kappas A. (1991) Comparative photoactivity of tin and zinc porphyrin inhibitors of heme oxygenase: pronounced photolability of the zinc compounds. Photochem Photobiol.; 54: 183-92.

Grunemar L, Ny L. (1997) Pitfalls using metalloporphyrins in carbon monoxide research. Trends Pharmacol Sci.; 18: 193-5.

Józkowicz A, Dulak J, Piatkowska E, Placha W, Dembinska-Kiec A. (2000) Ligands of peroxisome proliferator-activated receptor- $\gamma$ increase the generation of vascular endothelial growth factor in vascular smooth muscle cells and in macrophages. Acta Biochim Polon.; 47: 1147-57.

Józkowicz A, Huk I, Nigisch A, Weigel G, Weidinger F, Dulak J. (2002) Effect of prostaglandin- $\mathrm{J}_{2}$ on VEGF synthesis depends on the induction of heme oxygenase-1. Antioxidant Redox Signal.; 4: 577-85.

Kampfer H, Kolb N, Manderscheid M, Wetyler C, Pfeilschifter J, Frank S. (2001) Macrophage-derived heme-oxygenase-1: expression, regulation, and possible function in skin repair. Mol Med.; 7: 488-98.

Koh J, Suh SW, Gwag BJ, He Y, Hsu CY, Choi DW (1996) The role of zinc in selective neuronal death after transient global cerebral ischemia. Science.; 272: 1013-6.

Kreiser D, Nguen X, Wong R, Seidman D, Stevenson D, Quan S, Abraham N, Dennery P. (2002) Heme oxygenase modulates fetal growth in the rat. Lab Invest.; 82: 687-92.

Linden DJ, Narasimhan K, Gurfel D. (1993) Protoporphyrins modulate voltage-gated $\mathrm{Ca}$ current in AtT-20 pituitary cells. $J$ Neurophysiol.; 70: 2673-7. 
Luo D, Vincent SR. (1994) Metalloporphyrins inhibit nitric oxide-dependent cGMP formation in vivo. Eur J Pharmacol.; 267: 263-7.

Lutton JD, Abraham NG, Drummond GS, Levere RD, Kappas A. (1997) Zinc porphyrins: potent inhibitors of hematopoieses in animal and human bone marrow. Proc Natl Acad Sci U S A.; 94: 1432-6.

Marton LS, Wang X, Kowalczuk A, Zhang ZD, Windmeyer E, Macdonald RL. (2000) Effects of hemoglobin on heme oxygenase gene expression and viability of cultured smooth muscle cells. Am J Physiol Heart Circ Physiol.; 279: H2405-13.

Morse D, Choi AM. (2002) Heme oxygenase-1: the "emerging molecule" has arrived. Am J Respir Cell Mol Biol.; 27: 8-16.

Polte T, Oberle S, Schroder H. (1997) The nitric oxide donor SIN-1 protects endothelial cells from tumor necrosis factor-alpha-mediated cytotoxicity: possible role for cyclic GMP and heme oxygenase. J Mol Cardiol.; 29: 3305-10.

Polte T, Abate A, Dennery PA, Schroder H. (2000) Heme oxygenase-1 is a cGMP-inducible endothelial protein and mediates the cytoprotective action of nitric oxide. Arterioscler Thromb Vasc Biol.; 20: 1209-15.

Quato MK, Maines MD. (1985) Prevention of neonatal hyperbilirubinemia in non-human primates by Zn-protoporphyrin. Biochem J.; 226: $51-7$.

Regan RF, Guo Y, Kumar N. (2000) Heme oxygenase-1 induction protects murine cortical astrocytes from hemoglobin toxicity. Neurosci Lett.; 282: 1-4.

Sakaguchi S, lizuka Y, Furasawa S, Ishikawa M, Satoh S, Takayanagi M. (2002) Role of $\mathrm{Zn}(2+)$ in oxidative stress caused by endotoxin challenge. Eur J Pharmacol.; 451: 309-16.

Serfass L, Burstyn JN. (1998) Effect of heme oxygenase inhibitors on soluble guanylyl cyclase activity. Arch Biochem Biophys.; 359: $8-16$.

Snyder SH, Baranano DE. (2001) Heme oxygenase: a font with multiple messengers. Neuropsychopharmacology.; 25: 294-8.

Steffensrud S. (1998) Tin metalloporphyrins: an answer to neonatal jaundice. Neonatal Netw.; 17: $11-7$.

Tamion F, Richard V, Bonmarchand G, Leroy J, Lebreton JP, Thuillez C. (2001) Induction of heme-oxygenase-1 prevents the systemic responses to hemorrhagic shock. Am J Respir Crit Care Med.; 164: 1933-8.

Vreman HJ, Ekstrand BC, Stevenson DK. (1993) Selection of metalloporphyrin heme oxygenase inhibitors based on potency and photoreactivity. Pediatr Res.; 33: 195-200.

Vreman HJ, Cipkala DA, Stevenson DK. (1996) Characterization of porphyrin heme oxygenase inhibitors. Can J Physiol Pharmacol.; 74: 278-85.

Weiss G, Lutton JD, Fuchs D, Werner-Felmayer G, Bock G, Abraham NG, Kappas A, Levere RD, Wachter H. (1993) Comparative effects of heme and metalloporphyrins on interferon-gamma-mediated pathways in monocytic cells (THP-1). Proc Soc Exp Biol Med.; 202: 470-5.

White KA, Marletta MA. (1992) Nitric oxide synthase is a cytochrome $\mathrm{P}-450$ type hemoprotein. Biochemistry.; 31: 6627-31.

Wolff DJ, Naddelman RA, Lubeskie A, Saks DA. (1996) Inhibition of nitric oxide synthase isoforms by porphyrins. Arch Biochem Biophys.; 333: 27-34.

Zakhary R, Gaine SP, Dinerman JL, Ruat M, Flavahan NA, Snyder SH. (1996) Heme oxygenase-2: Endothelial and neuronal localization and role in endothelium-dependent relaxation. Proc Natl Acad Sci U S A.; 93: 795-8. 